Alma Mater Studiorum - Università di Bologna DEPARTMENT OF ECONOMICS

\title{
Information and Online Reviews
}

Oksana Loginova

Andrea Mantovani

Quaderni - Working Paper DSE N 996

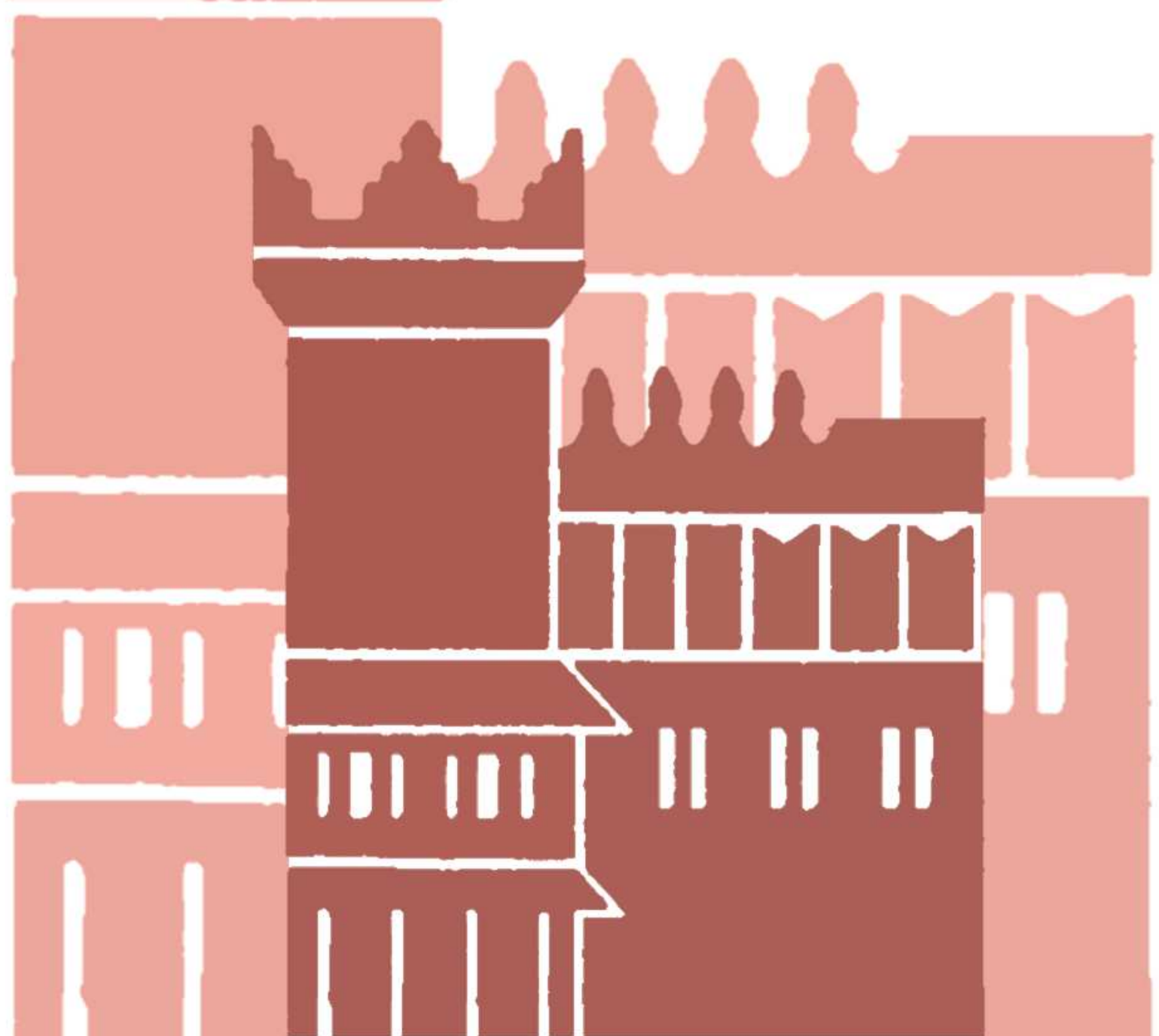




\title{
Information and Online Reviews
}

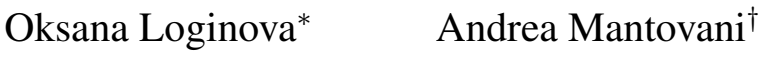

March 11, 2015

\begin{abstract}
Online review aggregators, such as TripAdvisor, HotelClub and OpenTable help consumers identify the products and services that best match their preferences. The goal of this study is to understand the impact of online review aggregators on firms and consumers. We adopt Salop's circular city model in which consumers initially do not know the locations of the firms in the product space. The firms decide whether or not to be listed on an online review aggregator's website and choose their prices. When a firm resorts to the aggregator, its location and price become observable to the consumers who visit the website. We consider two different scenarios, depending on the possibility for online firms to offer discounts to the consumers who book online. We show that in equilibrium not all firms will go online - some will remain offline. Online firms attract more customers than their offline counterparts due to reduced mismatch costs, but face a tougher price competition. Comparing the equilibrium prices, profits and the number of firms that go online across the scenarios, we derive interesting conclusions from the private and the social standpoints.
\end{abstract}

Keywords: online review aggregators, price discrimination, competition.

JEL codes: C72, D43, D61, L11, L13, M31.

\section{Introduction}

Recent years have been characterized by the flourishing of online sales. At the beginning, merchants created and used their own web pages both to increase their customer base and to facilitate sales to existing customers. Then, we observed the proliferation of e-commerce companies, web platforms, shopping websites and online auctions through which customers and merchants were able to buy and sell a broad variety of products and services worldwide (e.g., eBay, Amazon, Expedia). The next step was to gather consumers. Group buying became a common practice thanks to the extraordinary expansion of Groupon, followed by several other similar websites.

\footnotetext{
${ }^{*}$ Department of Economics, University of Missouri, 333 Professional Building, Columbia, MO 65211, USA; e-mail: loginovao@missouri.edu.

${ }^{\dagger}$ Department of Economics, University of Bologna, Strada Maggiore 45, 40125 Bologna, Italy, and Barcelona Institute of Economics (IEB), J. M. Keynes 1-11, 08034 Barcelona, Spain; e-mail: a mantovani@unibo. it.
} 
Each of these online selling methods has been analyzed in the economics and management literature, in order to understand the reasons of their success or failure, and their impact on market competition and welfare. ${ }^{1}$

However, a key player in this market has been partially neglected, at least by the mainstream theoretical literature: online review aggregators. ${ }^{2}$ They started to operate in the second part of 2000s and enjoyed a very rapid expansion ever since, becoming key players in hotel booking systems and restaurant reservation services, amongst others. We can distinguish review aggregators that are open, such as Booking.com and OpenTable, from those that are closed, such as Relais \& Chateaux and Gourmet Society. The latter are exclusive clubs which select their members based on income, education, and social status. ${ }^{3}$ Open aggregators are, obviously, more diffused, as they do not charge fees for final users. Aggregators also differ in the quality/amount of information they provide to customers. The source of information may be mainly left to consumer reviews (TripAdvisor, OpenTable, Urbanspoon), or it can be a combination of both the aggregator itself and peer reviews (Booking.com, TheFork, RatesToGo, HotelClub). In addition to information, some aggregators offer special services, such as personalized recommendations (Foursquare) and the possibility to book/purchase when the customer finds the desired product (Booking.com, RatesToGo, HotelClub, OpenTable, Gourmet Society, TheFork, Relais \& Chateaux). The latter service is particularly convenient in the lodging and catering sectors, for it allows to reduce consumer search costs by restricting the database to firms that are available (have a free room or a table) on the required day. Often, consumers who book online enjoy a discount, which could be either imposed by the aggregator or decided by the producer. Discounts are more frequent in closed aggregators. All restaurants that appear on Gourmet Society's website, for example, have agreed to offer a discount ( $25 \%$ off the total bill). On the contrary, restaurants listed on TheFork are free to decide their discounting policy (about 20\%$30 \%$ offer a lower price to their online clients). Several aggregators, however, prohibit price discrimination. Booking.com, for example, requires hotels to adhere to the rate parity policy by which they cannot offer rooms at lower prices to other online booking platforms, nor to those consumers who book through the hotel's website or show up at the hotel's lobby. Similarly, diners using OpenTable pay the same price as those who do not reserve through the aggregator.

The rise of review aggregators is strictly related to the persistence of asymmetric information in markets characterized by experience goods. Starting from the seminal contribution developed by Akerlof (1970), such asymmetries have been widely studied especially for the case of vertically differentiated goods. Insurances and warranties are commonly accepted solutions in the market for lemons. However, the same toolkit can not be easily implemented in the case of horizontally differentiated goods, as the perception of quality is subjective. Although firms often advertise feautures/characteristics of their product, consumers do not always rely on such a bias source of information. Partial solutions may be the brand's reputation in a specific market niche

\footnotetext{
${ }^{1}$ Seminal contributions by Alba et al. (1997) on consumer incentives to shift to new distribution channels and Bakos (1997) on electronic marketplaces that reduce search and increase competition among retailers have been followed by a proliferation of papers touching different aspects of online commerce. The literature on group buying, although pioneered by Che and Gale (1997), has only recently been developed, following the success of Groupon.

${ }^{2}$ In the next section we provide a brief description of the most relevant empirical papers that focus on the role of review aggregators.

${ }^{3}$ Membership is usually awarded through a combination of different channels such as private invitation from other members, interviews, and requires relatively expensive annual fees.
} 
and word-of-mouth. These remedies are not very effective in markets with unitary consumption, such as hotels, restaurants, or museums.

Our paper delivers a theoretical model to understand the impact of online review aggregators in horizontally differentiated markets. For exposition purposes only, through the paper we focus on aggregators that provide information and booking services in the catering sector, and we illustrate our model as a stylized version inspired by OpenTable or the Fork. ${ }^{4}$ They represent instructive examples of online services available to a wide range of consumers. The aggregator's website provides detailed information about restaurants, including location, type of cuisine, menu, prices, and dining atmosphere, thus helping the customer to narrow down her search to a few options that best match her tastes. Consumers rely on the aggregator, whose reputation is reinforced by the availability of peer reviews. ${ }^{5}$ The risk of fake reviews, although very interesting, is not the object of our paper.

Having such a case study in mind, we use a model of horizontal differentiation à la Salop (1979) to analyze the equilibrium behavior of consumers, restaurants and an online review aggregator. We assume that initially consumers do not know the locations of the restaurants in the product space. The restaurants first decide whether or not to be listed on the aggregator's website and then set their prices. When a restaurant goes online, its location and price become observable to the consumers that visit the website. Consumers who book a table through the aggregator are called e-users, while those who choose a restaurant in a more traditional way (yellow pages or randomly walking down the street) are called walkers. The proportion of eusers in the population is exogenously fixed. Finally, we assume that the aggregator charges restaurants a fixed fee. ${ }^{6}$

In the first setting that we consider, the aggregator prohibits restaurants to offer discounts to e-users. In the second, the aggregator allows restaurants to price discriminate between walkers and e-users. In both settings, we compute the equilibrium prices, profits, and the number of online restaurants. We show that in equilibrium not all restaurants will go online - some will remain offline. Online restaurants attract more customers than their offline counterparts due to reduced mismatch costs, but they face a tougher price competition. We find that in the first setting (uniform pricing), online restaurants charge a lower price than offline restaurants when the proportion of e-users is sufficiently high. In the second setting (price discrimination), online restaurants set the same price for walkers as offline restaurants, while offering a discount to e-users.

Although simple, our model allows us to derive interesting conclusions both from the private and the social standpoints. In particular, the analysis that we carry out brings forth three interesting results. First, for a given number of online restaurants, we find that allowing discounts may hurt the restaurants. Specifically, when the proportion of e-users in the population is

\footnotetext{
${ }^{4}$ TheFork operates in the restaurant market. It is currently available in France, Monaco, Spain, Belgium, Italy, and Switzerland under the names LaFourchette, ElTenedor and TheFork.

${ }^{5}$ OpenTable launched in 2008 the OpenTable Reviews Program, through which diners submit restaurant reviews and feedback. Only verified customers can write a review. On TheFork all customers are invited to leave a review (an average of $70 \%$ reviews per restaurant is currently available).

${ }^{6}$ In reality, some aggregators charge a fixed per-year fee (Urbanspoon), some a per-client/reservation fee (TheFork), and others a setup fee combined with a monthly fee and/or per-reservation fee (OpenTable). TripAdvisor charges businesses for clickable ads and business listings. See Moraga-Gonzalez and Wildenbeest (2012) for fees charged by shopbots, or comparison sites.
} 
relatively low, online restaurants are trapped in the prisoner's dilemma. Each restaurant has an incentive to offer a discount to capture more e-users; however, as they all offer a discount, the final price may be too low and the restaurants end up losing in comparison to uniform pricing.

Second, we consider the effect of price discrimination on the equilibrium number of online restaurants. In our theoretical framework, the social optimum is achieved when all restaurants go online. Hence, in order for discounting to be beneficial, it has to increase the number of online restaurants as compared to the uniform pricing setting. We find that discounting is socially preferable when the proportion of e-users is high and the fixed online fee is low. In this parameter region, price discrimination allows online restaurants to charge a higher price for walkers while profitably competing for e-users. As a result, more restaurants will choose to go online.

Third, we evaluate the aggregator's optimal policy and find that allowing discounts is never profitable for the aggregator, when the aggregator can select the online fee. Hence, potential conflicts of interest between the aggregator and the social planner may appear. In particular, following previous results, this happens when the proportion of e-users is relatively high. In addition, in such region it is more likely that the restaurants would profit from discounting. It would be, therefore, extremely important for the policy maker to intervene in order to guarantee an efficient solution.

The paper is structured as follows. In the next section we review the literature. The model is presented in Section 3. The two settings mentioned above are analyzed in Sections 4 and 5, respectively. In Section 6 we report the choice of the aggregator. Concluding remarks appear in Section 7.

\section{Related Literature}

Our paper contributes to explaining the private and social impacts of online selling when merchants resort to a review aggregator in order to disclose certain product features to final consumers. The role of review aggregators has so far received attention mainly in empirical studies. Ghose et al. (2012) show that consumers base their purchases on the available reviews. Anderson and Magruder (2012) suggest a positive relation between reviews and profits, thus confirming previous results on the effect of word-of-mouth (Chevalier and Mayzlin, 2006) and reviews by professional critics (Reinstein and Snyder, 2005; Hilger et al., 2011) on sales. Clearly, the effectiveness of online reviews depends on their credibility. ${ }^{7}$ In our model we abstract from the risk of fake reviews and assume that consumers trust the aggregator. ${ }^{8}$

We also extend the literature that examines the impact of online sales on offline prices. Empirical papers analyzing this issue are, among others, Brown and Goolsbee (2002) on individual insurance policies, and Morton et al. (2001) on pricing behavior of car dealerships. On the theoretical side, Lal and Sarvary (1999) and Zettelmeyer (2000) both consider firms compet-

\footnotetext{
${ }^{7}$ To increase their credibility, aggregators can allow only certified consumers to write a review. Brynjolfsson and Smith (2000) argue that branding awareness and trust are important sources of heterogeneity among Internet retailers. However, a 2012 Nielsen poll of more than 28000 Internet consumers from 56 countries revealed that online consumer reviews are the second most-trusted source of brand information after suggestions from friends and family.

${ }^{8}$ Dellarocas (2006), Mayzlin (2006) and Mayzlin et al. (2013), inter alia, study the phenomenon of manipulated reviews.
} 
ing through alternative distribution channels such as online shopping. They find that online shopping does not necessarily increase price competition. Similar results can be found in Loginova (2009), who provides a theoretical framework to study competition between online and offline retailers. She shows that there exist circumstances under which conventional stores may increase their prices when online rivals enter the market. One common feature of such theoretical models is that online shopping has pros and cons: on the one hand, it reduces travelling costs; on the other hand, consumers prefer to inspect a specific item before purchasing it. In our model, on the contrary, going online reduces mismatch costs for consumers without causing any discontent.

A stream of literature in economics and marketing has examined information disclosure in monopolistic and competitive settings. The seminal work of Lewis and Sappington (1994) considers a firm's incentive to reveal its private information to two different types of consumers in order to facilitate price discrimination. Anand and Shachar (2011) examine television networks which advertise their own show. This results in a trade-off as it increases the demand for some viewers, but reduces that for others. In our paper price discrimination between e-users and walkers may lead to a similar trade-off, although we focus mainly on price effects as demands remain unchanged. Recent papers by Sun (2010) and Gu and Xie (2013) study the incentives for producers to reveal multiple (vertical and horizontal) product attributes.

Our paper is also related to the practice of selective discounting, which provides opportunities for price discrimination. ${ }^{9}$ The conventional result is that price discrimination is privately adopted by producers and can be also welfare improving, as it allows to serve weaker markets which would otherwise be cut out (see Schmalensee, 1981; Varian, 1985; Stigler, 1987, inter alia). Regarding the specific case of third-degree price discrimination, it may decrease welfare when the demand function is linear (Aguirre et al., 2010; Aguirre, 2012). In our paper we consider a scenario in which the aggregator allows restaurants to price discriminate between e-users and walkers. Although the total quantity is constant regardless of the presence of the aggregator, the society at large may benefit if the number of firms going online increases when discounts are allowed. Edelman et al. (2014) examine the profitability and implications of online discount vouchers such as those used by Groupon. ${ }^{10}$ Their paper is pertinent to ours as such a pricing strategy facilitates price discrimination. They found that it pays off when merchants are patient and relatively unknown, given that discount vouchers also activate informative advertising. In our model, however, possible advertising effects are not taken into account.

Recent advances in technology opened up new opportunities for firms to implement innovative selling strategies (Shugan, 2004). One of these is group buying, whereby consumers obtain a discount if they group and achieve a required group size (see Che and Gale, 1997; Anand and Aron, 2003; and Jing and Xie, 2011). Other marketing strategies are referral reward programs (see Biyalogorsky et al., 2001), one-to-one promotion (Shaffer and Zhang, 2002) and advance selling (Xie and Shugan, 2001), among others. These papers explore very interesting issues which are related to our basic idea. They can provide opportunity for further research.

\footnotetext{
${ }^{9}$ This has been studied in seminal contributions by Varian (1980) and Narasimhan (1984, 1988), among others.

${ }^{10}$ Empirical surveys on Groupon are provided by Byers et al. (2011), Dholakia (2011), and Dholakia and Tsabar (2011), inter alia.
} 


\section{The Model}

The players in our model are uninformed consumers, restaurants and an online review aggregator. (Think of tourists visiting Paris and looking for a place to have dinner.) Consumers differ in their preferences for cuisine (French, Italian, Asian) and dining atmosphere (traditional, bistro, trendy, brasserie). We use Salop circular city to model consumer heterogeneity. Consumers of total mass one are uniformly distributed on a circle of circumference one. $N$ restaurants are located equidistantly around the circle; each produces a meal at the constant marginal cost $c$. When a consumer dines in a restaurant located at distance $x$ from him, his utility from consuming a meal (his valuation) is

$$
v-t x
$$

where $t$ represents the intensity of consumer tastes.

We assume that consumers do not know the locations of the restaurants nor their prices. Only when a consumer enters a restaurant, she observes its price, and only after the consumer has a meal there, she learns its location on the circle. Since the expected distance between the consumer and a randomly chosen restaurant is $1 / 4$, the consumer's expected utility equals $v-t / 4$. We also assume that as long as the restaurant's price does not exceed the consumer's expected utility, the consumer dines at that restaurant. (She does not check out other places for a lower price.) Thus, in equilibrium each restaurant will set its price equal to

$$
v-\frac{t}{4}
$$

leaving all consumers with zero expected payoffs.

We now introduce the online review aggregator, such as OpenTable, Urbanspoon, or TheFork. Suppose for a moment that all restaurants appear on the aggregator's website. This means that a consumer can visit the aggregator's website and learn the locations and prices of the $N$ restaurants. If all consumers visit the website, then we have Salop's circular city model (Salop, 1979). In equilibrium each restaurant will set its price equal to

$$
c+\frac{t}{N}
$$

To keep the equilibrium analysis as simple as possible, we assume that $v$ is sufficiently high,

$$
v-c>\frac{3 t}{4}
$$

This assumption guarantees that the consumer located halfway between two adjacent restaurants (having to travel $1 /(2 N)$ along the circle) receives a strictly positive payoff:

$$
v-\frac{t}{2 N}>c+\frac{t}{N}
$$

holds for any $N \geq 2$.

To make the model more realistic, we assume that only some consumers visit the aggregator's website. Let $\alpha \in[0,1]$ denote the fraction of consumers who find it worth their time and 


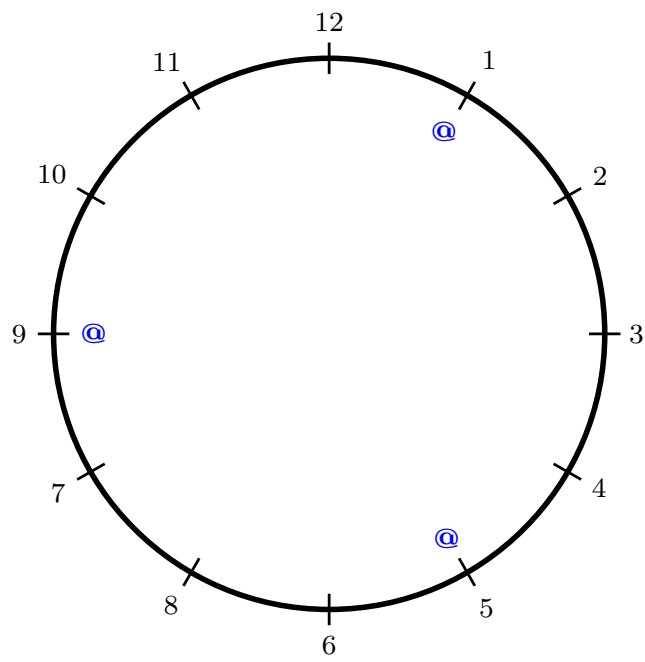

Figure 1: Online and Offline Restaurants on the Salop Circle

effort to check out the restaurants online. We will refer to these consumers as $e$-users, and to the rest (who choose a restaurant by simply walking down the street) as walkers.

Next, we assume that in order to appear on the aggregator's website, a restaurant should pay a fixed fee $k>0$ to the aggregator. Let $n$ denote the number of restaurants that go online (endogenously determined). Again, we assume that these $n$ restaurants are located equidistantly around the circle. In Figure 1, $N=12$ and $n=3$. Restaurants 1, 5 and 9 are on the aggregator's website, while the other restaurants are not. We will call the restaurants on the aggregator's website online restaurants and the rest - offline restaurants.

In the next section we study a setting in which online restaurants charge the same price to all their customers (walkers and e-users). In Section 5 we allow online restaurants to offer discounts to those consumers who book a table through the aggregator (i.e., to e-users).

\section{Setting I: Online Restaurants Charge Uniform Prices}

In this setting we assume that online restaurants do not price discriminate and charge the same price to walkers and e-users. Let $p_{\text {off }}^{*}$ be the price that offline restaurants charge in a symmetric Nash equilibrium, and let $p_{\text {on }}^{*}$ be the price of online restaurants. Since we have assumed that a walker dines at the first restaurant she walks in (as long as the price does not exceed his expected utility),

$$
p_{\text {off }}^{*}=v-\frac{t}{4} .
$$

Each of $N-n$ offline restaurants will attract $(1-\alpha) / N$ customers $(1 / N$ of all walkers $)$ and earn

$$
\pi_{\mathrm{off}}^{*}=\frac{1-\alpha}{N}\left(p_{\mathrm{off}}^{*}-c\right)=\frac{1-\alpha}{N}\left(v-\frac{t}{4}-c\right) .
$$


Now let us find $p_{\text {on }}^{*}$ and $\pi_{\text {on }}^{*}$. Suppose that there are at least two restaurants on the aggregator's website, $n \geq 2$, and $p_{\text {on }}^{*} \leq p_{\text {off }}^{*}=v-t / 4$ (proved in the Appendix). Then in equilibrium each online restaurant will attract $(1-\alpha) / N+\alpha / n$ customers $(1 / N$ of all walkers and $1 / n$ of all e-users).

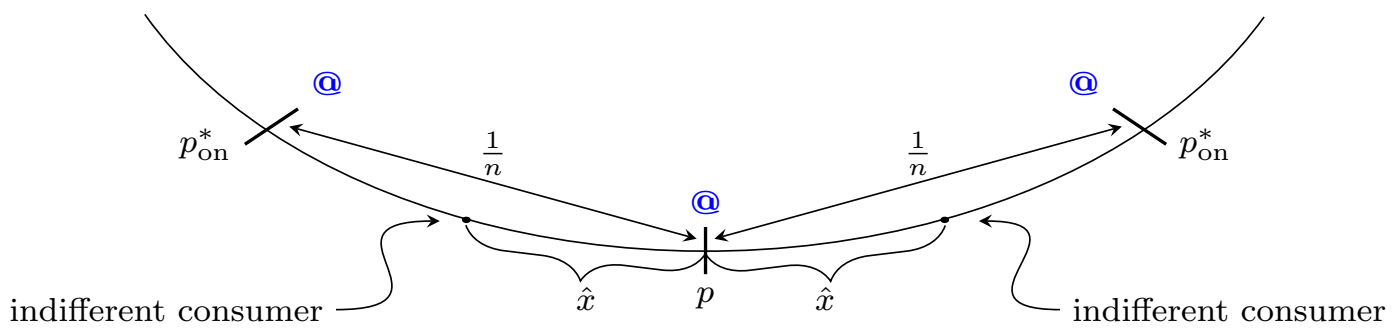

Figure 2: Determining the Equilibrium Price

Consider a deviation by an online restaurant from $p_{\text {on }}^{*}$ to $p$. The restaurant will attract $(1-$ $\alpha) / N+2 \alpha \hat{x}$ customers, where $\hat{x}$ is determined by

$$
\begin{gathered}
v-p-\hat{x} t=v-p_{\text {on }}^{*}-\left(\frac{1}{n}-\hat{x}\right) t, \\
\hat{x}=\frac{1}{2 n}+\frac{p_{\text {on }}^{*}-p}{2 t} .
\end{gathered}
$$

(See Figure 2.) Hence, the profit of this restaurant will be

$$
\left(\frac{1-\alpha}{N}+2 \alpha\left(\frac{1}{2 n}+\frac{p_{\mathrm{on}}^{*}-p}{2 t}\right)\right)(p-c) .
$$

The equilibrium requires

$$
p_{\mathrm{on}}^{*}=\arg \max _{p \leq v-\frac{t}{4}}\left(\frac{1-\alpha}{N}+\alpha\left(\frac{1}{n}+\frac{p_{\mathrm{on}}^{*}-p}{t}\right)\right)(p-c) .
$$

Differentiating the objective function with respect to $p$ and setting the derivative to zero leads to

$$
-\frac{\alpha}{t}(p-c)+\frac{1-\alpha}{N}+\alpha\left(\frac{1}{n}+\frac{p_{\mathrm{on}}^{*}-p}{t}\right)=0 .
$$

Substituting $p=p_{\text {on }}^{*}$ into the above equation and solving for $p_{\text {on }}^{*}$ yield

$$
p_{\mathrm{on}}^{*}=\min \left\{c+\frac{(1-\alpha) t}{\alpha N}+\frac{t}{n}, v-\frac{t}{4}\right\} .
$$

In equilibrium, each online restaurant earns

$$
\pi_{\mathrm{on}}^{*}=\left(\frac{1-\alpha}{N}+\frac{\alpha}{n}\right)\left(p_{\mathrm{on}}^{*}-c\right) .
$$

Thus, we have the following lemma. 
Lemma 1 (Prices and Profits in Setting I, for a Given Value of $n$ ). Consider Setting I and suppose there are $n$ online restaurants. Then in equilibrium each offline restaurant will charge

$$
p_{\mathrm{off}}^{*}=v-\frac{t}{4}
$$

and earn

$$
\pi_{\mathrm{off}}^{*}=\frac{1-\alpha}{N}\left(v-\frac{t}{4}-c\right) .
$$

Online restaurants will set their prices equal to

$$
p_{\mathrm{on}}^{*}(n)= \begin{cases}c+\frac{(1-\alpha) t}{\alpha N}+\frac{t}{n} & \text { if } \alpha \geq \alpha_{0}(n) \\ v-\frac{t}{4} & \text { if } \alpha<\alpha_{0}(n),\end{cases}
$$

earning

$$
\pi_{\mathrm{on}}^{*}(n)= \begin{cases}\left(\frac{1-\alpha}{N}+\frac{\alpha}{n}\right)^{2} \frac{t}{\alpha} & \text { if } \alpha \geq \alpha_{0}(n) \\ \left(\frac{1-\alpha}{N}+\frac{\alpha}{n}\right)\left(v-\frac{t}{4}-c\right) & \text { if } \alpha<\alpha_{0}(n),\end{cases}
$$

where

$$
\alpha_{0}(n)=\frac{t}{t+N\left(v-\frac{t}{4}-\frac{t}{n}-c\right)} .
$$

The results of this lemma are intuitive. When more consumers visit the aggregator's website, $\alpha \geq \alpha_{0}(n)$, the e-users become the primary target for online restaurants. This leads the restaurants to charge

$$
p_{\mathrm{on}}^{*}(n)=c+\frac{(1-\alpha) t}{\alpha N}+\frac{t}{n}<v-\frac{t}{4} .
$$

Ceteris paribus, $\alpha$ is more likely to fall into the region $\left[\alpha_{0}(n), 1\right]$ for larger values of $n\left(\alpha_{0}^{\prime}(n)<\right.$ $0)$. As $n$ increases, $p_{\text {on }}^{*}(n)$ decreases.

On the contrary, when $\alpha<\alpha_{0}(n)$, the walkers become the primary target for online restaurants, and they charge

$$
p_{\mathrm{on}}^{*}=v-\frac{t}{4} .
$$

This is more likely to happen when $n$ is small.

Next, we find how many restaurants will go online in equilibrium. Recall that in order to appear on the aggregator's website, a restaurant should pay $k$ to the aggregator. Hence, the equilibrium number of online restaurants is determined by

$$
\pi_{\mathrm{off}}^{*}=\pi_{\mathrm{on}}^{*}(n)-k .
$$

The equilibrium profit of an offline restaurant, $\pi_{\text {off }}^{*}$, does not depend on $n$, while the equilibrium profit of an online restaurant, $\pi_{\text {on }}^{*}(n)$, decreases in $n$ and approaches $\pi_{\text {off }}^{*}$ as $n \rightarrow \infty$ (see the proof of Proposition 1 in the Appendix). Thus, (5) has a unique solution as long as $k$ is not prohibitively large. 
Proposition 1 (Equilibrium Number of Online Restaurants in Setting I). In Setting I, the equilibrium number of online restaurants is given by

$$
n^{*}= \begin{cases}N & \text { if } k \leq k_{1} \\ \frac{\alpha}{\sqrt{\left(k+\frac{1-\alpha}{N}\left(v-\frac{t}{4}-c\right)\right) \frac{\alpha}{t}}-\frac{1-\alpha}{N}} & \text { if } k \in\left(k_{1}, k_{2}\right] \\ \frac{\alpha\left(v-\frac{t}{4}-c\right)}{k} & \text { if } k>k_{2},\end{cases}
$$

where

$$
k_{1}=\frac{\alpha\left(v-\frac{t}{4}-c\right)}{N}-\max \left\{\frac{v-\frac{t}{4}-c}{N}-\frac{t}{N^{2} \alpha}, 0\right\}
$$

and

$$
k_{2}=\frac{\alpha\left(v-\frac{t}{4}-c\right)}{N}+\max \left\{\frac{\alpha\left(v-\frac{t}{4}-c\right)^{2}}{t}-\frac{v-\frac{t}{4}-c}{N}, 0\right\} .
$$

Observe that when

$$
\begin{gathered}
\alpha \leq \frac{t}{N\left(v-\frac{t}{4}-c\right)}, \\
\max \left\{\frac{\alpha\left(v-\frac{t}{4}-c\right)^{2}}{t}-\frac{v-\frac{t}{4}-c}{N}, 0\right\}=\max \left\{\frac{v-\frac{t}{4}-c}{N}-\frac{t}{N^{2} \alpha}, 0\right\}=0 .
\end{gathered}
$$

Hence, $k_{1}=k_{2}$ and the set $\left(k_{1}, k_{2}\right]$ is empty for these values of $\alpha$. When

$$
\alpha>\frac{t}{N\left(v-\frac{t}{4}-c\right)},
$$

$k_{1}<k_{2}$. While $k_{2}$ is always positive, $k_{1}$ may be negative. If this happens, then $k \leq k_{1}$ in the above proposition is an empty set. That is, even when the fixed online fee is zero, not all restaurants will choose to go online.

Recall that our equilibrium analysis goes through when there are at least two online restaurants, $n \geq 2$. This assumption imposes an upper limit to the value of $k$. It follows from Proposition 1 that $n^{*} \geq 2$ if and only if

$$
k \leq \frac{\alpha}{2}\left(v-\frac{t}{4}-c\right) .
$$

Finally, we find the socially optimal number of online restaurants and compare it with $n^{*}$. The socially optimal number, denoted by $n^{\text {so }}$, minimizes the mismatch costs incurred by the e-users:

$$
\min _{n} \alpha \frac{t}{4 n} \text {. }
$$

(As $k$ represents a transfer from an online restaurant to the aggregator, it is not a cost to the society. That is why $n k$ does not enter the objective function.) Hence,

$$
n^{\mathrm{so}}=N \text {. }
$$

While efficiency requires all restaurants to go online, in equilibrium $n^{*} \leq N$, with strict inequality when $k>k_{1}$. The private and the social interests are, therefore, not aligned when $k$ is sufficiently high. 


\section{Setting II: Online Restaurants Charge Different Prices to Walk- ers and E-users}

Suppose that online restaurants can offer discounts to those consumers who book a table through the aggregator. It is easy to see that in equilibrium they will charge walkers the price

$$
\bar{p}_{\mathrm{on}}^{\dagger}=v-\frac{t}{4}
$$

and e-users

$$
\underline{p}_{\mathrm{on}}^{\dagger}(n)=c+\frac{t}{n}
$$

This implies the discount of

$$
v-\frac{t}{4}-\frac{t}{n}-c
$$

which is positive for all $n \geq 2$ due to the assumption (1). Thus, in equilibrium each online restaurant earns the profit

$$
\pi_{\mathrm{on}}^{\dagger}(n)=\frac{1-\alpha}{N}\left(\bar{p}_{\mathrm{on}}^{\dagger}-c\right)+\frac{\alpha}{n}\left(\underline{p}_{\mathrm{on}}^{\dagger}-c\right)=\frac{1-\alpha}{N}\left(v-\frac{t}{4}-c\right)+\frac{\alpha t}{n^{2}} .
$$

Lemma 2 (Equilibrium Prices and Profits in Setting II, for a Given Value of $n$ ). Consider Setting II and suppose there are $n$ online restaurants. Then the equilibrium prices and profits are given by:

$$
\begin{gathered}
p_{\text {off }}^{\dagger}=\bar{p}_{\text {on }}^{\dagger}=v-\frac{t}{4}, \\
\underline{p}_{\text {on }}^{\dagger}(n)=c+\frac{t}{n}, \\
\pi_{\text {off }}^{\dagger}=\frac{1-\alpha}{N}\left(v-\frac{t}{4}-c\right),
\end{gathered}
$$

and

$$
\pi_{\mathrm{on}}^{\dagger}(n)=\frac{1-\alpha}{N}\left(v-\frac{t}{4}-c\right)+\frac{\alpha t}{n^{2}}
$$

Let us compare the equilibrium prices and profits of online restaurants across the two settings for a given value of $n$. In Setting I, an online restaurant serves $(1-\alpha) / N+\alpha / n$ customers at price $p_{\text {on }}^{*}$. In Setting II, it serves $(1-\alpha) / N$ customers at price $\underline{p}_{\text {on }}^{\dagger}$ and $\alpha / n$ customers at price $\bar{p}_{\text {on }}^{\dagger}$. When $\alpha<\alpha_{0}(n)$,

$$
\underline{p}_{\mathrm{on}}^{\dagger}(n)<p_{\mathrm{on}}^{*}(n)=\bar{p}_{\mathrm{on}}^{\dagger}
$$

which immediately implies

$$
\pi_{\text {on }}^{*}(n)>\pi_{\text {on }}^{\dagger}(n) .
$$

In other words, discounting hurts online restaurants. When $\alpha \geq \alpha_{0}(n)$,

$$
\underline{p}_{\text {on }}^{\dagger}(n)<p_{\text {on }}^{*}(n)<\bar{p}_{\text {on }}^{\dagger}
$$


and the comparison between $\pi_{\mathrm{on}}^{*}(n)$ and $\pi_{\mathrm{on}}^{\dagger}(n)$ can go in either direction. Specifically,

$$
\pi_{\mathrm{on}}^{*}(n)=\left(\frac{1-\alpha}{N}+\frac{\alpha}{n}\right)^{2} \frac{t}{\alpha}>\pi_{\mathrm{on}}^{\dagger}(n)=\frac{1-\alpha}{N}\left(v-\frac{t}{4}-c\right)+\frac{\alpha t}{n^{2}}
$$

if and only if

$$
\alpha<\alpha_{1}(n)=\frac{t}{t+N\left(v-\frac{t}{4}-\frac{2 t}{n}-c\right)} .
$$

Since $\alpha_{1}(n)>\alpha_{0}(n)$ for any value of $n$, we have the following result.

Lemma 3 (Comparing Equilibrium Profits of Online Restaurants in Settings I and II, for a Given Value of $n$ ). Suppose there are $n$ online restaurants. The restaurants lose from discounting, $\pi_{\text {on }}^{*}(n)>\pi_{\text {on }}^{\dagger}(n)$, when $\alpha<\alpha_{1}(n)$.

We would like to stress that in the above lemma the number of online restaurants is fixed. Consider Setting I and assume that there are $n$ online restaurants. Now suppose that the aggregator allows discounting. Lemma 3 indicates that the ability to price discriminate between walkers and e-users benefits online restaurants only when $\alpha$ - the fraction of e-users in the population - is sufficiently high. Low values of $\alpha$ give rise to the prisoner's dilemma. We demonstrate its existence in the next two paragraphs. Specifically, we prove that an online restaurant has a unilateral incentive to charge different prices to walkers and e-users, provided that the other online restaurants do not price discriminate.

In the absence of discounts, the equilibrium prices and profits are as in Lemma 1. If we allow one restaurant to price discriminate, will it choose to do so? In the Appendix we show that when $\alpha \geq \alpha_{0}(n)$, the deviating restaurant will charge walkers $v-t / 4$ and e-users

$$
p^{\mathrm{dev}}=c+\frac{(1-\alpha) t}{2 \alpha N}+\frac{t}{n}<p_{\mathrm{on}}^{*}=c+\frac{(1-\alpha) t}{\alpha N}+\frac{t}{n},
$$

thereby increasing its profit. However, when all online restaurants are allowed to price discriminate, they charge e-users

$$
\underline{p}_{\text {on }}^{\dagger}=c+\frac{t}{n}<p^{\mathrm{dev}} .
$$

When $\alpha \in\left(\alpha_{0}, \alpha_{1}\right)$, online restaurants lose compared to Setting I where discounts are not allowed.

We also show in the Appendix that when $\alpha<\alpha_{0}(n)$, the deviating restaurant will charge walkers $v-t / 4$ and e-users

$$
p^{\mathrm{dev}}=\frac{c+v}{2}-\frac{t}{8}+\frac{t}{2 n} .
$$

We verify that, again,

$$
\underline{p}_{\text {on }}^{\dagger}=c+\frac{t}{n}<p^{\mathrm{dev}}<p_{\mathrm{on}}^{*}=v-\frac{t}{4} .
$$

The result is, as before, intuitive. As more online restaurants adopt the discount strategy for eusers, the price that e-users pay drops, and the restaurants get trapped in the prisoner's dilemma for all $\alpha<\alpha_{0}(n)$. 
Next, we calculate the equilibrium number of online restaurants:

$$
\begin{gathered}
\pi_{\text {off }}^{\dagger}=\pi_{\text {on }}^{\dagger}(n)-k, \\
\frac{1-\alpha}{N}\left(v-\frac{t}{4}-c\right)=\frac{1-\alpha}{N}\left(v-\frac{t}{4}-c\right)+\frac{\alpha t}{n^{2}}-k, \\
n^{\dagger}=\min \left\{\sqrt{\frac{\alpha t}{k}}, N\right\} .
\end{gathered}
$$

Proposition 2 (Equilibrium Number of Online Restaurants in Setting II). In Setting II, the equilibrium number of online restaurants is given by

$$
n^{\dagger}= \begin{cases}N & \text { if } k \leq \frac{\alpha t}{N^{2}} \\ \sqrt{\frac{\alpha t}{k}} & \text { if } k>\frac{\alpha t}{N^{2}}\end{cases}
$$

We see that $n^{\dagger} \leq n^{\text {so }}=N$, with strict inequality for $k>\alpha t / N^{2}$. Put differently, the social welfare is maximized only when the fixed cost $k$ is sufficiently small, $k \leq \alpha t / N^{2}$. Hence, also in this setting, the private and the social interests fail to coincide when $k$ is sufficiently high.

It is straightforward to show that $n^{\dagger} \geq 2$ if and only if

$$
k \leq \frac{\alpha t}{4} \text {. }
$$

Comparing this condition with (6), we notice that

$$
\frac{\alpha t}{4}<\frac{\alpha}{2}\left(v-\frac{t}{4}-c\right)
$$

holds under the assumption (1). The maximum value of $k$ compatible with the equilibrium number of online restaurants being at least two in both settings is, therefore,

$$
k_{\max }=\frac{\alpha t}{4} .
$$

For the rest of our analysis we will assume that $k$ does not exceed $k_{\text {max }}$.

Finally, we compare the equilibrium number of online restaurants in this setting with that in Setting I. It immediately follows from Propositions 1 and 2 that when $k \leq \min \left\{\alpha t / N^{2}, k_{1}\right\}$, $n^{\dagger}=n^{*}=n^{\text {so }}=N$. When $k>\min \left\{\alpha t / N^{2}, k_{1}\right\}$, either $n^{*}<n^{\dagger} \leq n^{\text {so }}$ or $n^{\dagger}<n^{*} \leq n^{\text {so }}$. The first (second) case implies that allowing (prohibiting) discounts is beneficial for the society.

Note that

$$
\frac{\alpha}{\sqrt{\left(k+\frac{1-\alpha}{N}\left(v-\frac{t}{4}-c\right)\right) \frac{\alpha}{t}-\frac{1-\alpha}{N}}}>\sqrt{\frac{\alpha t}{k}}
$$

(the left-hand side is the equilibrium number of online restaurants in Setting I when $k \in\left(k_{1}, k_{2}\right]$ and the right-hand side is the equilibrium number of online restaurants in Setting II when $k>$ $\alpha t / N^{2}$ ) if and only if

$$
k>\frac{t}{4 \alpha}\left(\frac{\alpha\left(v-\frac{t}{4}-c\right)}{t}-\frac{1-\alpha}{N}\right)^{2}=k_{3},
$$


and

$$
\frac{\alpha\left(v-\frac{t}{4}-c\right)}{k}>\sqrt{\frac{\alpha t}{k}}
$$

(the left-hand side the equilibrium number of online restaurants in Setting I when $k>k_{2}$ and the right-hand side is the equilibrium number of online restaurants in Setting II when $k>\alpha t / N^{2}$ ) always holds under the assumption (1) and for $k \leq k_{\max }$.

Tedious algebra (see the Appendix) reveals that the thresholds $k_{1}, k_{2}$ and $\alpha t / N^{2}$ and the critical value $k_{3}$ (obtained above) line up as

$$
k_{3}<\frac{\alpha t}{N^{2}}<k_{1} \leq k_{2}
$$

when

$$
\alpha<\frac{t}{N\left(v-\frac{t}{4}-c-\frac{t}{N}\right)}=\alpha_{2}
$$

and as

$$
k_{1}<\frac{\alpha t}{N^{2}}<k_{3}<k_{2}
$$

when $\alpha>\alpha_{2}$. Thus, we have the following proposition.

Proposition 3 (Equilibrium Number of Online Restaurants in Settings I and II and Efficiency). Let $\alpha<\alpha_{2}$. In this case the socially optimal number of online restaurants is reached in both settings when

$$
k \leq \frac{\alpha t}{N^{2}}
$$

Otherwise, prohibiting discounts is beneficial (provides a higher number of online restaurants).

When $\alpha>\alpha_{2}$, the socially optimal number of online restaurants is reached in both settings when $k \leq k_{1}$. For high values of $k, k>k_{3}$, prohibiting discounts is beneficial. On the contrary, allowing discounts is beneficial for intermediate values of $k, k \in\left(k_{1}, k_{3}\right)$.

Figure 3 provides a useful representation of the above results. ${ }^{11}$ Apart from the case in which the fee is very low, $k<\min \left\{k_{1}, \alpha t / N^{2}\right\}$, the equilibrium number of restaurants that decide to go online depends on the interplay between $\alpha$ and $k$. Consider the admissible parametric region in which $k \leq k_{\max }$. If the fraction of e-users is relatively low, then uniform pricing (Setting I) allows more restaurants to go online when $k>\alpha t / N^{2}$. Moreover, for $k \in\left(\alpha t / N^{2}, k_{1}\right)$, the efficient solution is reached, as $\left(n^{\dagger}<\right) n^{*}=n^{\text {so }}$. The intuition goes as follows. From the discussion after Lemma 3 we know that online restaurants lose from price discrimination, as they get trapped in the prisoner's dilemma. A lower number of restaurants would, therefore, be able to afford the fee for being listed on the aggregator's website. On the contrary, for relatively high values of $\alpha$, online restaurants can charge higher prices for walkers while at the same time competing for e-users (their main target). In this region discounting is socially preferred, as more restaurants can afford the fee to go online. Notice also that, when $k \in\left(\max \left\{0, k_{1}\right\}, \alpha t / N^{2}\right)$, the efficient outcome is guaranteed under price discrimination, as $\left(n^{*}<\right) n^{\dagger}=n^{\text {so }}$. Finally, observe that the higher is the proportion of e-users in the population, the larger is the region where discounting is socially preferred.

\footnotetext{
${ }^{11}$ In drawing the picture, we fixed the following parameter values: $v=2, c=0, t=1$, and $N=12$.
} 


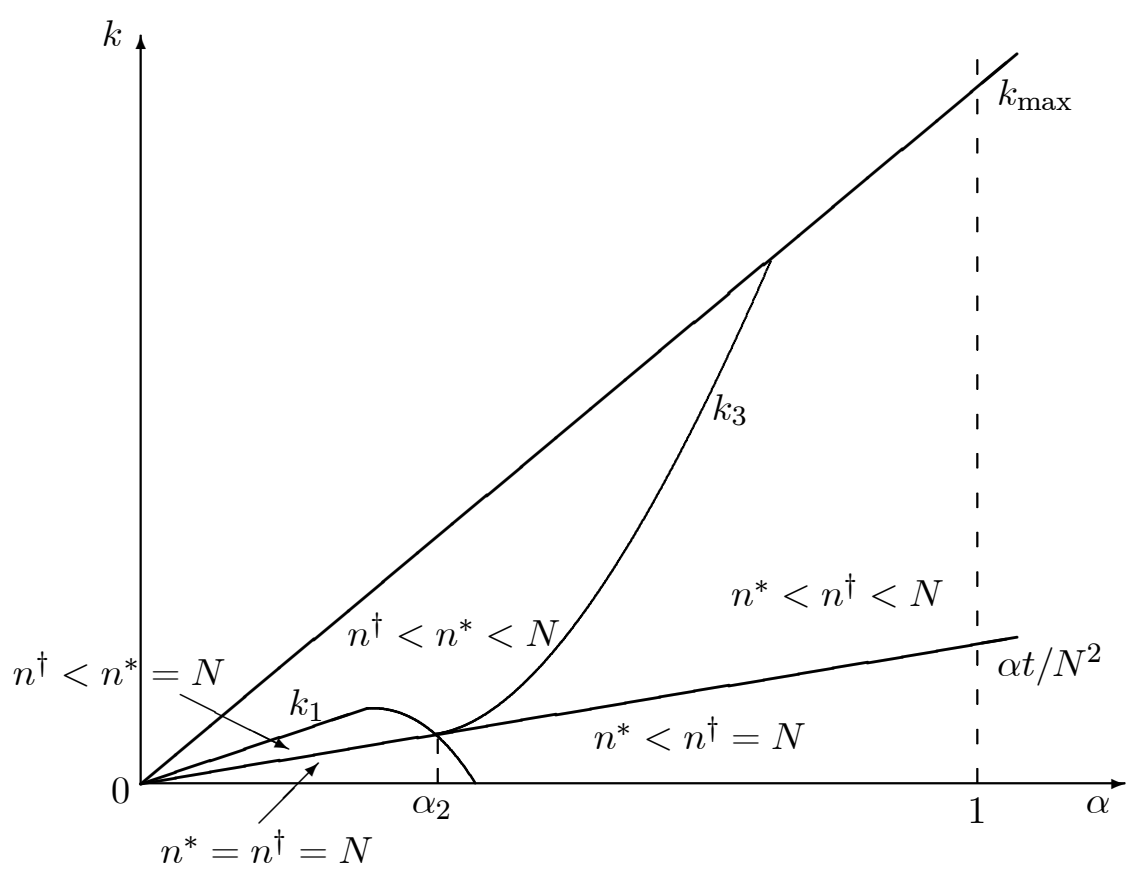

Figure 3: Equilibrium and Efficient Number of Restaurants

\section{The Aggregator's Optimal Policy}

In this section we make the aggregator an active player. Suppose for a moment that the aggregator can decide whether to prohibit (Setting I) or to allow discounts (Setting II). If the aggregator's profit is simply the product of the equilibrium number of online restaurants and the fee $k$, then it will choose the setting that yields the highest number of online restaurants.

To make our model more realistic, we assume that the aggregator not only decides whether to allow discounts, but also chooses the online fee $k$. Will the aggregator's optimal policy result in $N$ restaurants (the socially optimal number) going online? Consider Setting I. The aggregator's profit is $k n^{*}$, where $n^{*}$ is a decreasing function of $k$. As it was noted right after Proposition 1,

$$
\alpha<\frac{t}{N\left(v-\frac{t}{4}-c\right)}
$$

implies

$$
k_{1}=k_{2}=\frac{\alpha\left(v-\frac{t}{4}-c\right)}{N} .
$$

If this is the case, then

$$
k n^{*}(k)= \begin{cases}k N & \text { if } k \leq k_{1} \\ \alpha\left(v-\frac{t}{4}-c\right) & \text { if } k>k_{1}\end{cases}
$$


Obviously, the aggregator's profit attains its maximum,

$$
\alpha\left(v-\frac{t}{4}-c\right)
$$

at any $k \geq k_{1}$. Note that $k=k_{1}$ induces all restaurants to go online, $n^{*}\left(k_{1}\right)=N$. This is socially optimal.

When

$$
\alpha>\frac{t}{N\left(v-\frac{t}{4}-c\right)},
$$

$k_{1}<k_{2}$, so

$$
k n^{*}(k)= \begin{cases}k N & \text { if } k \leq k_{1} \\ \frac{k \alpha}{\sqrt{\left(k+\frac{1-\alpha}{N}\left(v-\frac{t}{4}-c\right)\right) \frac{\alpha}{t}}-\frac{1-\alpha}{N}} & \text { if } k \in\left(k_{1}, k_{2}\right] \\ \alpha\left(v-\frac{t}{4}-c\right) & \text { if } k>k_{2} .\end{cases}
$$

It is easy to show that $k_{2}$ yields a higher profit to the aggregator than $k_{1}$. Indeed,

$$
\alpha\left(v-\frac{t}{4}-c\right)>k_{1} N=\alpha\left(v-\frac{t}{4}-c\right)-N \max \left\{\frac{v-\frac{t}{4}-c}{N}-\frac{t}{N^{2} \alpha}, 0\right\},
$$

which holds as the term

$$
\max \left\{\frac{v-\frac{t}{4}-c}{N}-\frac{t}{N^{2} \alpha}, 0\right\}
$$

is strictly positive for

$$
\alpha>\frac{t}{N\left(v-\frac{t}{4}-c\right)} .
$$

Therefore, $k_{1}$, which leads to the socially optimal number of online restaurants, will not be chosen by the aggregator.

In Setting II, the aggregator's profit is

$$
k n^{\dagger}(k)= \begin{cases}k N & \text { if } k \leq \frac{\alpha t}{N^{2}} \\ \sqrt{\alpha t k} & \text { if } k>\frac{\alpha t}{N^{2}}\end{cases}
$$

It is easy to see that $k n^{\dagger}(k)$ is increasing in $k$, which immediately implies that the optimal $k$ is $k_{\max }=\alpha t / 4$. The aggregator's maximum profit in Setting II equals

$$
\frac{\alpha t}{2}
$$

This is lower than what the aggregator can get in Setting I: the inequality

$$
\frac{\alpha t}{2}<\alpha\left(v-\frac{t}{4}-c\right)
$$

always holds due to the assumption (1).

We summarize the above results in the following proposition. 
Proposition 4 (The Aggregator's Optimal Policy). When it comes to shaping its policy, the aggregator will prohibit discounts. The fee chosen by the aggregator will induce all restaurants to go online when

$$
\alpha<\frac{t}{N\left(v-\frac{t}{4}-c\right)} .
$$

For other values of $\alpha$, the optimal $k$ will result in less than the socially optimal number of online restaurants.

When the aggregator chooses the fee $k$, it faces a trade-off, as increasing the fee reduces the number of restaurants that can afford to go online. In such a case, prohibiting discounts is preferred by the aggregator as it allows to charge a sufficiently high fee without losing too many restaurants. Proposition 4 indicates that when the proportion of e-users is low, the aggregator will choose $k$ that induces all restaurants to go online. Allowing discounts is less profitable to the aggregator as the number of restaurants that could afford to go online decreases more rapidly than under uniform pricing.

This has relevant policy implications, as the interests of the aggregator may not always be aligned with those of the society. In particular, when the proportion of e-users is relatively high, imposing uniform pricing implies that a lower number of restaurants would go online, as we can deduce from Proposition 3 (or Figure 3). In addition, for relatively high values of $\alpha$, the restaurants are more likely to gain when adopting price discrimination. In such an interval region, as a consequence, the social planner should intervene to restore price discrimination.

\section{Concluding Remarks}

In this paper we used a model of horizontal product differentiation à la Salop (1979) to analyze the impact of an online review aggregator on the equilibrium behavior of consumers and producers in a market with asymmetric information. In particular, we imagined a situation where uninformed consumers (tourists visiting a new city) are looking for a place to eat. The aggregator was assumed to provide accurate information about the restaurants listed on its website (online restaurants), therefore helping consumers to find their best match. The population of consumers was divided into those willing to spend time on the Internet (e-users) and those enjoying roaming around the city while looking for a place to eat (walkers).

We considered two different settings, depending on whether online restaurants can offer discounts to e-users. We showed that in the first setting (that of uniform pricing), online restaurants charge a lower price than their offline counterparts only when the proportion of e-users is sufficiently high. In the second setting, in which discounting is allowed, we found that e-users always pay less than walkers. For a given number of online restaurants, we highlighted that the profitability of price discrimination crucially depends on the composition of consumers. If the population of consumers is mainly composed by walkers, then online restaurants do not gain from discounting, as they end up being trapped in the prisoner's dilemma. As compared to uniform pricing, they cannot increase the price for walkers, but they are pressured by competition to offer a discount to e-users. On the contrary, price discrimination pays off when the proportion of e-users is relatively high. This was the first important finding of our analysis. 
Our paper also provided interesting predictions on the social desirability of allowing discounting. We computed the equilibrium number of online restaurants in both settings and compared it with the first best for the society, which in our model simply requires all restaurants to go online. We showed that, apart from the case in which the fee charged by the aggregator is very small, it is again the composition of consumers that plays a major role. When walkers predominate, then uniform pricing allows more restaurants to go online, as under discounting online restaurants are trapped in the prisoner's dilemma. On the contrary, when the proportion of e-users is relatively high, then online restaurants gain from price discrimination, so a higher number of restaurants can afford to pay the fee for being listed online. Hence, discounting is socially preferred. In both cases the first best can be reached when the fee charged by the aggregator is sufficiently low.

Finally, we considered the aggregator's optimal policy regarding the fee and whether to impose uniform pricing or to allow discounts for e-users. We discovered that the aggregator prefers uniform pricing as it can increase the fee without losing too many subscribers (online restaurants). However, this revealed potential areas of conflict between the aggregator and the social planner. In particular, when e-users predominate, imposing uniform pricing implies that a lower number of restaurants can afford to go online, and this has obvious negative consequences in terms of social welfare.

Throughout the paper we used the catering sector for exposition purposes. The results of our model are applicable to the lodging sector, where the role of review aggregators is more and more prominent. ${ }^{12}$ Related to our last result, recent concerns have been raised in the EU regarding anticompetitive effects of the rate parity policy adopted by hotel booking portals such as Booking.com, Expedia, and HRS. The German competition authority alleged that the rate parity policy imposed by HRS virtually eliminated competition for low-end (cheaper) rooms, thus reducing the number of hotels in the market. ${ }^{13}$ In France, hotel unions accused Booking.com of using the price parity clause to prevent entry of alternative reservation platforms. After an intervention of the French competition authority, Booking.com removed the price parity clause from its contracts. ${ }^{14}$ Competition regulators in Italy, UK and Sweden echoed such concerns. In the UK, the Office of Fair Trading (OFT) accepted in 2014 formal commitments from Booking.com and Expedia Inc. (Expedia) to allow online travel agents and hotels to offer discounts on rates for hotel rooms. ${ }^{15}$ In the U.S., a class action lawsuit has been filed against Expedia, Orbitz, Travelocity, Marriott, Starwood, InterContinental, Hilton and other major online travel agencies (OTAs) and hotel chains for price fixing. ${ }^{16}$

The analysis carried out in this paper relied on many simplifying assumptions. First of all, we considered the presence of only one aggregator, and supposed that it conveys reliable information at almost zero cost for consumers. This can be justified by the fact that consumers tend to resort to just a few trusted sources of information, those who successfully win the race to

\footnotetext{
${ }^{12}$ In the EU, following a survey conducted by Coach Omnium, $93 \%$ of consumers use the Internet to find a hotel, and $70 \%$ of online hotel bookings are made through hotel booking platforms.

${ }^{13}$ Bundeskartellamt continues to view HRS's best price clause critically, see http://www.bundeskartellamt.de/SharedDocs/Meldung/EN/Pressemitteilungen/2013/25_07_2013_HRS.html

${ }^{14}$ See Authorité de la Concurrence, République Française, Press Release, 15 December 2014: Online Hotel Booking Sector.

${ }^{15}$ See http://www.oft.gov.uk/OFTwork/competition-act-and-cartels/ca98/closure/online-booking

${ }^{16}$ See Nikita Turik, et al. v. Expedia, Inc., et al., C.A. No. 3:1204365 Northern District of Texas, 2012.
} 
become the reference points for consumers unfamiliar with certain product characteristics. For this reason, aggregators usually do not charge final users, but compete for rents coming from the firms that want to get online visibility. Therefore, introducing competition among aggregators would result in lower fees being charged to firms, without changing the qualitative results of our analysis.

A second limitation of our model is that it focuses only on uninformed consumers. In an extension of the basic setting, we introduced local consumers, who previously had the opportunity to try different restaurants in the city. ${ }^{17}$ Once a local consumer finds a restaurant that satisfies her tastes, she dines only there, provided that the price does not exceed her valuation. Assuming a proportion $\beta$ of locals in the population of consumers, we found that our results are still valid. Indeed, by substituting the fraction $\alpha$ of e-users with the fraction of e-users among the population of tourists, i.e. $\tilde{\alpha} \equiv \alpha(1-\beta)$, one can notice that it is possible to reproduce the whole analysis carried out in the paper.

A third criticism that can be made to our model is that it assumed a fixed fraction of e-users in the population of consumers. Although very recent, the proliferation of online reviews is not a new phenomenon. Consumers already got used to online information provided by review aggregators. We are mainly interested in the consequences of allowing or not discounts in terms of how many merchants can afford to go online rather than in the rise of online aggregators.

We also acknowledge that our analysis has been performed under some specific parametric conditions. However, such conditions have been always justified not only for algebraic tractability, but also for being well suited to the specific market case that we wanted to study. All in all, we are also convinced that the basic model that we provided allows to capture the impact of online aggregators in a simple but significant way.

\section{References}

[1] Aguirre, I., 2012. Welfare Effects of Third Degree Price Discrimination: Ippolito Meets Schmalensee and Varian, in R. Laratta (ed) Social Welfare, InTech, ISBN 978-953-510208-3. Graphic Analysis of Third-Degree Price Discrimination.

[2] Aguirre, I., Cowan, S., Vickers, J., 2010. Monopoly Price Discrimination and Demand Curvature. American Economic Review, 100, 1601-15.

[3] Akerlof, G.A., 1970. The Market for 'Lemons': Qualitative Uncertainty and the Market Mechanism. Quarterly Journal of Economics, 84, 488-500

[4] Alba, J., Lynch, J., Weitz, B., Janiszewski, C., Lutz, R., Sawyer, A., Wood, S., 1997. Interactive Home Shopping: Consumer, Retailer and Manufacturer Incentives to Participate in Electronic Marketplaces. Journal of Marketing, 61, 38-53.

[5] Anand, B. N., Shachar, R., 2011. Advertising, the Matchmaker. RAND Journal of Economics, 42, 205-245.

\footnotetext{
${ }^{17}$ The extension with local consumers is available upon request.
} 
[6] Anand, K. S., Aron, R., 2003. Group Buying on the Web: A Comparison of PriceDiscovery Mechanisms. Management Science, 49, 1546-1562.

[7] Anderson, M., Magruder, J., 2012. Learning from the Crowd: Regression Discontinuity Estimates of the Effects of an Online Review Database. The Economic Journal, 122, 957989.

[8] Bakos, J. Y., 1997. Reducing Buyer Search Costs: Implications for Electronic Marketplaces. Management Science, 43, 1676-1692.

[9] Biyalogorsky, E., Gerstner, E., Libai, B., 2001. Customer Referral Management: Optimal Reward Programs. Marketing Science, 20, 82-95.

[10] Brynjolfsson, E., Smith, M., 2000. Frictionless Commerce? A Comparison of Internet and Conventional Retailers. Management Science, 46, 563-585.

[11] Brown, J. R., Goolsbee, A., 2002. Does the Internet Make Markets More Competitive? Evidence from the Life Insurance Industry. Journal of Political Economy, 110, 481-507.

[12] Byers, J. W., Mitzenmacher, M., Potamias, M., Zervas, G., 2011. A Month in the Life of Groupon. Mimeo.

[13] Che, Y.-K., Gale, I., 1997. Buyer Alliances and Managed Competition. Journal of Economics \& Management Strategy, 6, 175-200.

[14] Chevalier, J. A., Mayzlin, D., 2006. The Effect of Word of Mouth on Sales: Online Book Reviews. Journal of Marketing Research, 43, 345-354.

[15] Dellarocas, C., 2006. Strategic Manipulation of Internet Opinion Forums: Implications for Consumers and Firms. Management Science, 52, 1577-1593.

[16] Dholakia, U. M., 2011. How effective are Groupon promotions for businesses? Mimeo, Rice University.

[17] Dholakia, U. M., Tsabar, G., 2011. A startup's experience with running a Groupon promotion. Mimeo, Rice University.

[18] Ghose, A., Ipeirotis, P. G., Li, B., 2012. Designing Ranking Systems for Hotels on Travel Search Engines by Mining User-Generated and Crowdsourced Content. Marketing Science, 31, 493-520.

[19] Edelman, B., Jaffe, S., Kominers, S. D., 2014. To Groupon or not to Groupon: The Profitability of Deep Discounts. Harvard Business School Working Paper 11-063.

[20] Gu, Z., Xie, J., 2013. Facilitating Fit Revelation in the Competitive Market. Management Science, 59, 1196-1212.

[21] Hilger, J., Rafert, G., Villas-Boas, S., 2011. Expert Opinion and the Demand for Experience Goods: An Experimental Approach in the Retail Wine Market. Review of Economics and Statistics, 93, 1289-1296. 
[22] Jing, X., Xie, J., 2011. Group Buying: A New Mechanism for Selling Through Social Interactions. Management Science, 57, 1354-1372.

[23] Lal, R., Sarvary, M., 1999. When and How is the Internet Likely to Decrease Price Competition? Marketing Science, 18, 485-503.

[24] Lewis, T., Sappington, D., 1994. Supplying Information to Facilitate Price Discrimination. International Economic Review, 35, 309-327.

[25] Loginova, O., 2009. Real and Virtual Competition. Journal of industrial Economics, 57, 319-342.

[26] Mayzlin, D., 2006. Promotional Chat on the Internet. Marketing Science, 25, 155-163.

[27] Mayzlin, D., Dover, Y., Chevalier, J., 2013. Promotional Reviews: An Empirical Investigation of Online Review Manipulation. American Economic Review, 104, 2421-55.

[28] Moraga-Gonzalez, J. L., Wildenbeest, M. R., 2012. Comparison Sites, in Martin Peitz and Joel Waldfogel (editors), Handbook of the Digital Economy, Oxford University Press, 224-53.

[29] Morton, S. F.; Zettelmeyer, F., Silva-Risso, J., 2001. Internet Car Retailing. Journal of Industrial Economics, 49, 501-519.

[30] Narasimhan, C., 1984. A Price Discrimination Theory of Coupons. Marketing Science, 3, 128-147.

[31] Narasimhan, C., 1988. Competitive Promotional Strategies. The Journal of Business, 61, 427-49.

[32] Nielsen: Global Consumers' Trust in 'Earned' Advertising Grows in Importance, April 10, 2012, www.nielsen.com.

[33] Reinstein, D. A., Snyder, C. M., 2005. The Influence of Expert Reviews on Consumer Demand for Experience Goods: a Case Study of Movie Critics. Journal of Industrial Economics, 53, 27-51.

[34] Salop, S. C., 1979. Monopolistic Competition with Outside Goods. Bell Journal of Economics, 10, 141-156.

[35] Schmalensee, R., 1981. Output and Welfare Implications of Monopolistic Third Degree Price Discrimination. American Economic Review, 71 (1), 242-47.

[36] Shaffer, G., Zhang, Z. J., 2002. Competitive One-to-One Promotions. Management Science, 48, 1143-1160.

[37] Shugan, S. M., 2004. The Impact of Advancing Technology on Marketing and Academic Research. Marketing Science, 23, 469-475.

[38] Stigler, G. J., 1987. The theory of price. Macmillan USA. 
[39] Sun, M., 2010. Disclosing Multiple Product Attributes. Journal of Economics \& Management Strategy, 20, 195 - 224.

[40] Varian, H. R., 1980. A Model of Sales. American Economic Review, 70, 651-59.

[41] Varian, H. R., 1985. Price Discrimination and Social Welfare. American Economic Review, 75, 870-875.

[42] Xie, J., Shugan, S. M., 2001. Electronic Tickets, Smart Cards, and Online Prepayments: When and How to Advance Sell. Marketing Science, 20, 219-243.

[43] Zettelmeyer, F., 2000. Expanding to the Internet: Pricing and Communications Strategies When Firms Compete on Multiple Channels. Journal of Marketing Research, 37, 292-308.

\section{Appendix}

\section{Proof of Lemma 1.}

In the derivations preceding Lemma 1 we have guessed that $p_{\mathrm{on}}^{*} \leq v-t / 4$. That is, the price that the online restaurants charge in equilibrium does not repel walkers. We also did not check deviations to prices above $v-t / 4$.

1. First, we will show that whenever

$$
p_{\mathrm{on}}^{*}=v-\frac{t}{4},
$$

an online restaurant will not benefit from deviating to a price $p \in(v-t / 4, v]$. Indeed, the deviating restaurant will only attract $2 \alpha \hat{x}$ customers, where

$$
\hat{x}=\frac{1}{2 n}+\frac{v-\frac{t}{4}-p}{2 t} .
$$

Hence, its profit will be

$$
2 \alpha\left(\frac{1}{2 n}+\frac{v-\frac{t}{4}-p}{2 t}\right)(p-c)
$$

Differentiating with respect to $p$ and setting the derivative to zero yields

$$
\begin{gathered}
-\frac{1}{t}(p-c)+\frac{1}{n}+\frac{v-t / 4-p}{t}=0, \\
p=\frac{c+\frac{t}{n}+v-\frac{t}{4}}{2} .
\end{gathered}
$$

Observe that this price is below $v-t / 4$ :

$$
\frac{c+\frac{t}{n}+v-\frac{t}{4}}{2}<v-\frac{t}{4},
$$




$$
v-c>\frac{t}{n}+\frac{t}{4}
$$

which holds for any $n \geq 2$ under the assumption (1). This confirms that deviations to prices above $v-t / 4$ are not profitable.

2. Next, we will show that the equilibrium price $p_{\mathrm{on}}^{*}$ cannot exceed $v-t / 4$. Suppose the contrary, $p_{\mathrm{on}}^{*}>v-t / 4$. The walkers are not served by the online restaurants, hence

$$
p_{\mathrm{on}}^{*}=c+\frac{t}{n} \text {. }
$$

However,

$$
c+\frac{t}{n}<v-\frac{t}{4}
$$

holds for any $n \geq 2$ under the assumption (1). This contradicts the supposition.

3. For the threshold $\alpha_{0}(n)$ we solve

$$
\begin{gathered}
c+\frac{(1-\alpha) t}{\alpha N}+\frac{t}{n}=v-\frac{t}{4}, \\
\alpha_{0}(n)=\frac{t}{t+N\left(v-\frac{t}{4}-\frac{t}{n}-c\right)} .
\end{gathered}
$$

Observe that $\alpha_{0}(n)<1$, as

$$
v-\frac{t}{4}-\frac{t}{n}-c>0
$$

for all $n \geq 2$ due to the assumption (1).

\section{Proof of Proposition 1.}

That $\pi_{\mathrm{on}}^{*}(n)$ decreases in $n$ can be easily seen from (3) and (4). Indeed, since

$$
c+\frac{(1-\alpha) t}{\alpha N}+\frac{t}{n}
$$

decreases in $n$,

$$
p_{\mathrm{on}}^{*}(n)=\min \left\{c+\frac{(1-\alpha) t}{\alpha N}+\frac{t}{n}, v-\frac{t}{4}\right\}
$$

decreases in $n$. Next, because both

$$
\frac{1-\alpha}{N}+\frac{\alpha}{n}
$$

and

$$
p_{\mathrm{on}}^{*}(n)-c
$$

decrease in $n$,

$$
\pi_{\mathrm{on}}^{*}(n)=\left(\frac{1-\alpha}{N}+\frac{\alpha}{n}\right)\left(p_{\mathrm{on}}^{*}(n)-c\right)
$$

decreases in $n$. The rest of the proof is completed in three steps: 
1. If $k$ is small,

$$
k \leq \pi_{\mathrm{on}}^{*}(N)-\pi_{\mathrm{off}}^{*}= \begin{cases}\frac{\alpha\left(v-\frac{t}{4}-c\right)}{N}+\frac{t}{N^{2} \alpha}-\frac{v-\frac{t}{4}-c}{N} & \text { if } \alpha \geq \frac{t}{N\left(v-\frac{t}{4}-c\right)} \\ \frac{\alpha\left(v-\frac{t}{4}-c\right)}{N} & \text { if otherwise, }\end{cases}
$$

then $n^{*}=N$.

2. If $k>\pi_{\mathrm{on}}^{*}(N)-\pi_{\mathrm{off}}^{*}$, the equilibrium number of restaurants is determined by

$$
\frac{1-\alpha}{N}\left(v-\frac{t}{4}-c\right)=\left(\frac{1-\alpha}{N}+\frac{\alpha}{n}\right)^{2} \frac{t}{\alpha}-k
$$

for large values of $\alpha$, so

$$
n^{*}=\frac{\alpha}{\sqrt{\left(k+\frac{1-\alpha}{N}\left(v-\frac{t}{4}-c\right)\right) \frac{\alpha}{t}}-\frac{1-\alpha}{N}} .
$$

If $\alpha$ is small, (5) becomes

$$
\frac{1-\alpha}{N}\left(v-\frac{t}{4}-c\right)=\left(\frac{1-\alpha}{N}+\frac{\alpha}{n}\right)\left(v-\frac{t}{4}-c\right)-k
$$

hence

$$
n^{*}=\frac{\alpha\left(v-\frac{t}{4}-c\right)}{k} .
$$

The threshold value for $\alpha$ can be found from

$$
\begin{gathered}
\frac{\alpha}{\sqrt{\left(k+\frac{1-\alpha}{N}\left(v-\frac{t}{4}-c\right)\right) \frac{\alpha}{t}-\frac{1-\alpha}{N}}}=\frac{\alpha\left(v-\frac{t}{4}-c\right)}{k}, \\
\alpha=\frac{t+\frac{t N k}{v-\frac{t}{4}-c}}{t+N\left(v-\frac{t}{4}-c\right)} .
\end{gathered}
$$

Thus, we have

$$
n^{*}= \begin{cases}\frac{\alpha}{\sqrt{\left(k+\frac{1-\alpha}{N}\left(v-\frac{t}{4}-c\right)\right) \frac{\alpha}{t}-\frac{1-\alpha}{N}}} & \text { if } \alpha \geq \frac{t+\frac{t N k}{v-\frac{t}{4}-c}}{t+N\left(v-\frac{t}{4}-c\right)} \\ \frac{\alpha\left(v-\frac{t}{4}-c\right)}{k} & \text { if } \alpha<\frac{t+\frac{t N k}{v-\frac{t}{4}-c}}{t+N\left(v-\frac{t}{4}-c\right)}\end{cases}
$$

or

$$
n^{*}= \begin{cases}\frac{\alpha}{\sqrt{\left(k+\frac{1-\alpha}{N}\left(v-\frac{t}{4}-c\right)\right) \frac{\alpha}{t}}-\frac{1-\alpha}{N}} & \text { if } k \leq \frac{\alpha\left(v-\frac{t}{4}-c\right)}{N}+\frac{\alpha\left(v-\frac{t}{4}-c\right)^{2}}{t}-\frac{v-\frac{t}{4}-c}{N} \\ \frac{\alpha\left(v-\frac{t}{4}-c\right)}{k} & \text { if } k>\frac{\alpha\left(v-\frac{t}{4}-c\right)}{N}+\frac{\alpha\left(v-\frac{t}{4}-c\right)^{2}}{t}-\frac{v-\frac{t}{4}-c}{N} .\end{cases}
$$


3. Combining the results in steps 1 and 2 yields:

$$
n^{*}= \begin{cases}N & \text { if } k \leq \frac{\alpha\left(v-\frac{t}{4}-c\right)}{N}+\frac{t}{N^{2} \alpha}-\frac{v-\frac{t}{4}-c}{N} \\ \frac{\alpha}{\sqrt{\left(k+\frac{1-\alpha}{N}\left(v-\frac{t}{4}-c\right)\right) \frac{\alpha}{t}-\frac{1-\alpha}{N}}} & \text { if } k \in\left(\frac{\alpha\left(v-\frac{t}{4}-c\right)}{N}+\frac{t}{N^{2} \alpha}-\frac{v-\frac{t}{4}-c}{N},\right. \\ & \left.\frac{\alpha\left(v-\frac{t}{4}-c\right)}{N}+\frac{\alpha\left(v-\frac{t}{4}-c\right)^{2}}{t}-\frac{v-\frac{t}{4}-c}{N}\right] \\ \frac{\alpha\left(v-\frac{t}{4}-c\right)}{k} & \text { if } k>\frac{\alpha\left(v-\frac{t}{4}-c\right)}{N}+\frac{\alpha\left(v-\frac{t}{4}-c\right)^{2}}{t}-\frac{v-\frac{t}{4}-c}{N}\end{cases}
$$

if

$$
\alpha \geq \frac{t}{N\left(v-\frac{t}{4}-c\right)}
$$

and

$$
n^{*}= \begin{cases}N & \text { if } k \leq \frac{\alpha\left(v-\frac{t}{4}-c\right)}{N} \\ \frac{\alpha\left(v-\frac{t}{4}-c\right)}{k} & \text { if } k>\frac{\alpha\left(v-\frac{t}{4}-c\right)}{N}\end{cases}
$$

if

$$
\alpha<\frac{t}{N\left(v-\frac{t}{4}-c\right)}
$$

\section{Derivation of (7).}

When $\alpha \geq \alpha_{0}(n)$,

$$
p_{\mathrm{on}}^{*}=c+\frac{(1-\alpha) t}{\alpha N}+\frac{t}{n} .
$$

The deviating restaurant will charge walkers $v-t / 4$ and e-users

$$
p^{\mathrm{dev}}=\arg \max _{p \leq v-\frac{t}{4}}\left(\frac{1-\alpha}{N}\left(v-\frac{t}{4}-c\right)+2 \alpha \hat{x}(p-c)\right)
$$

where $\hat{x}$ is determined by

$$
\begin{gathered}
v-p-\hat{x} t=v-p_{\mathrm{on}}^{*}-\left(\frac{1}{n}-\hat{x}\right) t \\
\hat{x}=\frac{1}{2 n}+\frac{p_{\mathrm{on}}^{*}-p}{2 t}=\frac{1}{2 n}+\frac{c+\frac{(1-\alpha) t}{\alpha N}+\frac{t}{n}-p}{2 t} .
\end{gathered}
$$

That is,

$$
p^{\mathrm{dev}}=\arg \max _{p \leq v-\frac{t}{4}}\left(\frac{1-\alpha}{N}\left(v-\frac{t}{4}-c\right)+\frac{2 \alpha}{t}\left(\frac{c}{2}+\frac{(1-\alpha) t}{2 \alpha N}+\frac{t}{n}-\frac{p}{2}\right)(p-c)\right) .
$$

Differentiating

$$
\left(\frac{c}{2}+\frac{(1-\alpha) t}{2 \alpha N}+\frac{t}{n}-\frac{p}{2}\right)(p-c)
$$

with respect to $p$ and setting the derivative to zero yield

$$
p^{\mathrm{dev}}=c+\frac{(1-\alpha) t}{2 \alpha N}+\frac{t}{n} .
$$




\section{Derivation of $(8)$.}

When $\alpha<\alpha_{0}(n)$,

$$
p_{\mathrm{on}}^{*}=v-\frac{t}{4} .
$$

The deviating restaurant will charge walkers $v-t / 4$ and e-users

$$
p^{\mathrm{dev}}=\arg \max _{p \leq v-\frac{t}{4}}\left(\frac{1-\alpha}{N}\left(v-\frac{t}{4}-c\right)+2 \alpha \hat{x}(p-c)\right),
$$

where

$$
\hat{x}=\frac{1}{2 n}+\frac{p_{\mathrm{on}}^{*}-p}{2 t}=\frac{1}{2 n}+\frac{v-\frac{t}{4}-p}{2 t} .
$$

That is,

$$
p^{\mathrm{dev}}=\arg \max _{p \leq v-\frac{t}{4}}\left(\frac{1-\alpha}{N}\left(v-\frac{t}{4}-c\right)+\frac{2 \alpha}{t}\left(\frac{v}{2}-\frac{t}{8}+\frac{t}{2 n}-\frac{p}{2}\right)(p-c)\right) .
$$

Differentiating

$$
\left(\frac{v}{2}-\frac{t}{8}+\frac{t}{2 n}-\frac{p}{2}\right)(p-c)
$$

with respect to $p$ and setting the derivative to zero yield

$$
p^{\mathrm{dev}}=\frac{c+v}{2}-\frac{t}{8}+\frac{t}{2 n} .
$$

\section{Proof of (9) and (10).}

The proof is completed in five steps:

1. First, we show that $k_{2}<k_{3}$ for all values of $\alpha$. When

$$
\begin{aligned}
& \alpha<\frac{t}{N\left(v-\frac{t}{4}-c\right)}, \\
& k_{2}=\frac{\alpha\left(v-\frac{t}{4}-c\right)}{N} .
\end{aligned}
$$

The inequality $k_{2}<k_{3}$ can be written as

$$
\frac{\alpha\left(v-\frac{t}{4}-c\right)}{N}<\frac{\alpha\left(v-\frac{t}{4}-c\right)^{2}}{t},
$$

or

$$
v-c>\frac{t}{4}+\frac{t}{N},
$$

which holds for $N \geq 2$ under the assumption (1). When

$$
\alpha>\frac{t}{N\left(v-\frac{t}{4}-c\right)},
$$




$$
k_{2}=\frac{\alpha\left(v-\frac{t}{4}-c\right)}{N}+\left(\frac{\alpha\left(v-\frac{t}{4}-c\right)^{2}}{t}-\frac{v-\frac{t}{4}-c}{N}\right) .
$$

The inequality $k_{2}<k_{3}$, obviously, holds.

2. Next, we show that $k_{1}=\alpha t / N^{2}$ if and only if $\alpha=\alpha_{2}$. Indeed, when

$$
\begin{gathered}
\alpha<\frac{t}{N\left(v-\frac{t}{4}-c\right)}, \\
k_{1}=\frac{\alpha_{2}\left(v-\frac{t}{4}-c\right)}{N} .
\end{gathered}
$$

It is easy to see that the equation $k_{1}=\alpha t / N^{2}$ does not have a solution. When

$$
\begin{gathered}
\alpha>\frac{t}{N\left(v-\frac{t}{4}-c\right)}, \\
k_{1}=\frac{\alpha_{2}\left(v-\frac{t}{4}-c\right)}{N}-\left(\frac{v-\frac{t}{4}-c}{N}-\frac{t}{N^{2} \alpha_{2}}\right) .
\end{gathered}
$$

Solving $k_{1}=\alpha t / N^{2}$ yields

$$
\begin{gathered}
\frac{\alpha\left(v-\frac{t}{4}-c\right)}{N}-\left(\frac{v-\frac{t}{4}-c}{N}-\frac{t}{N^{2} \alpha}\right)=\frac{\alpha t}{N^{2}}, \\
\frac{\left(1-\alpha^{2}\right) t}{N^{2} \alpha}=\frac{(1-\alpha)\left(v-\frac{t}{4}-c\right)}{N}, \\
\frac{(1+\alpha) t}{N \alpha}=v-\frac{t}{4}-c, \\
\alpha=\frac{t}{N\left(v-\frac{t}{4}-c-\frac{t}{N}\right)}=\alpha_{2} .
\end{gathered}
$$

3. It immediately follows from the previous step that $k_{1}>\alpha t / N^{2}$ when $\alpha<\alpha_{2}$ and $k_{1}<$ $\alpha t / N^{2}$ when $\alpha>\alpha_{2}$.

4. It is left to show that $k_{4}=\alpha t / N^{2}$ if and only if $\alpha=\alpha_{2}$. We have:

$$
\begin{gathered}
\frac{t}{4 \alpha}\left(\frac{\alpha\left(v-\frac{t}{4}-c\right)}{t}-\frac{1-\alpha}{N}\right)^{2}=\frac{\alpha t}{N^{2}}, \\
\left(\frac{\alpha\left(v-\frac{t}{4}-c\right)}{t}-\frac{1-\alpha}{N}\right)^{2}=\frac{4 \alpha^{2}}{N^{2}} \\
\frac{\alpha\left(v-\frac{t}{4}-c\right)}{t}-\frac{1-\alpha}{N}=\frac{2 \alpha}{N} \\
\alpha=\frac{t}{N\left(v-\frac{t}{4}-c-\frac{t}{N}\right)}=\alpha_{2} .
\end{gathered}
$$

5. It immediately follows from the previous step that $k_{4}<\alpha t / N^{2}$ when $\alpha<\alpha_{2}$ and $k_{4}>$ $\alpha t / N^{2}$ when $\alpha>\alpha_{2}$. 


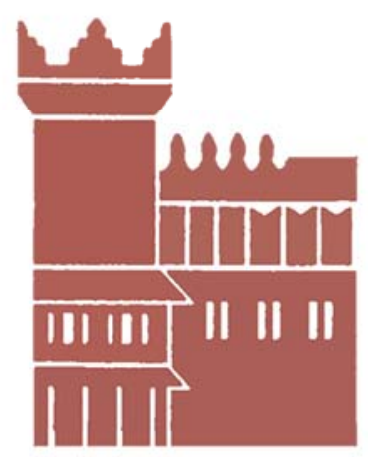

Alma Mater Studiorum - Università di Bologna DEPARTMENT OF ECONOMICS

Strada Maggiore 45

40125 Bologna - Italy

Tel. +39051 2092604

Fax +390512092664

http://www.dse.unibo.it 